\title{
Optimization of magnetic amplification by flow constraints in turbulent liquid sodium
}

\author{
M.D. Nornberg, ${ }^{*}$ N.Z. Taylor, and C.B. Forest \\ University of Wisconsin-Madison \\ 1150 University Ave. \\ Madison, WI 53706 and
}

Center for Magnetic-Self Organization in Laboratory and Astrophysical Plasmas

\author{
K. Rahbarnia \\ Max-Planck-Institut fur Plasmaphysik \\ Wendelsteinstr. 1, D-17491 \\ Greifswald, Germany \\ E. Kaplan \\ Helmholtz-Zentrum Dresden-Rossendorf \\ P.O. Box 510119, D-01314 \\ Dresden, Germany
}

(Dated: February 19, 2014) 


\begin{abstract}
Direct measurements of the vector turbulent emf in a driven two-vortex flow of liquid sodium were performed in the Madison Dynamo Experiment ${ }^{1}$. The measured turbulent emf is anti-parallel with the mean current and is almost entirely described by an enhanced resistivity which increases the threshold for a kinematic dynamo. We have demonstrated that this enhanced resistivity can be mitigated by eliminating the largest-scale eddies through the introduction of baffles. By tailoring the flow to reduce large-scale components and control the helical pitch we have reduced the power required to drive the impellers, doubled the magnetic flux generated by differential rotation, and increased the decay time of externally-applied magnetic fields. Despite these improvements, the flows remain sub-critical to the dynamo instability due to the reemergence of turbulent fluctuations at high flow speeds.
\end{abstract}

PACS numbers: 47.65.-d, 47.35.Tv, 52.72.+v, 47.35.-i

Keywords: dynamo, turbulent resistivity, mean field theory, quasi-linear theory, turbulence, MHD 


\section{INTRODUCTION}

One of the defining characteristics of turbulence is its propensity to enhance diffusion. In hydrodynamics, turbulence enhances the diffusion of particles and heat (passive scalars) and momentum (a vector field). The vortex tube stretching inherent in turbulent flows facilitates the transfer of both energy and vorticity to progressively smaller scales in the inertial range of a turbulent spectrum of fluctuations ${ }^{2}$. Large velocity gradients develop at sufficiently small scale that they are efficiently smoothed out by viscosity. Despite the weakness of viscous transport on the large-scale flow, the turbulent cascade provides an efficient mechanism for quickly directing the energy flow to progressively smaller scales where it is dissipated.

In the case of magnetohydrodynamic turbulence in kinetically dominated flows $\left(\rho v^{2} \gg\right.$ $\left.B^{2} / \mu_{0} \rho\right)$, the magnetic field is also advected by the turbulent eddies. In the kinematic regime where the Lorentz force (or back reaction) associated with flow-generated magnetic fields is assumed to be weak, the electric field induced by turbulent eddies can be approximated by first-order effects $^{3,4}$. For isotropic turbulence, two effects dominate: the induction of magnetic field by helical flows and the diffusion of magnetic flux. This flux diffusion, in direct analogy to turbulent diffusion of a passive scalar, is invoked in astrophysical context, for example, whenever direct numerical simulations are used to model stellar dynamos ${ }^{5}$. To perform solar and stellar dynamo simulations with available resources they must be conducted with diffusivities enhanced far above levels of resistive and viscous diffusion ${ }^{6}$. Typically dynamical models assume negligible resistive diffusion compared with turbulent diffusion.

The turbulent enhancement of resistive diffusion is also quite important when considering the design of a laboratory experiment that uses shear flow to excite MHD instabilities like the dynamo ${ }^{7-10}$. The requirement to provide sufficient shear to stretch and amplify magnetic field lines results in flows at fluid Reynolds numbers sufficiently high to excite hydrodynamic instabilities. The presence of hydrodynamic turbulence in the kinematic phase of a dynamo can either enhance the flux amplification if the eddies are sufficiently helical or enhance flux diffusion through the above-mentioned mechanism akin to turbulent mixing.

In this paper, we report a series of experiments conducted to determine the effect of hydrodynamic turbulence on the kinematic phase of flow liquid sodium whose mean-flow is designed to self-excite and become an MHD dynamo. We interrogate the turbulent flow by applying external fields to measure the amplification of magnetic flux at increasing flow speeds. We also pulse the 
applied field to determine the time it takes to decay with the expectation that it will increase in duration as the flow speed is increased and nears the threshold for self-generation of magnetic field. We make a series of changes to the boundary conditions of the flow to specifically (1) reduce the largest possible correlation length of the velocity fluctuations and (2) optimize the pitch of the flow. We find that the amplification of a magnetic field applied along the direction of the dominant kinematic eigenmode of the mean flow increases with each added flow constraint. We also find that the pulse decay time for the same applied field increases as well. However, these improvements saturate at higher flow speeds before the flow achieves self-excitation of a dynamo apparently due to nonlinear transfer of energy out of the dominant mode due to the reemergence of turbulent fluctuations.

\section{TURBULENT LIQUID METAL FLOWS}

Strongly turbulent flows are generated in the Madison Dynamo Experiment which consists of a $0.53 \mathrm{~m}$ radius sphere filled with liquid sodium at a temperature between $98-120^{\circ} \mathrm{C}$ (see Fig. 1$)^{11}$. Two counter-rotating impellers have been designed to drive a twin-vortex flow optimized for a low threshold for exciting a magnetohydrodynamic dynamo. Liquid sodium has the same density and kinematic viscosity as that of water at near room temperature; the flow speeds required to excite a dynamo are at fluid Reynolds numbers of $R e \sim 10^{7}$. This particular shear flow is hydrodynamically unstable at $R e \sim 300^{12}$ and so at these flow speeds the flow consists of fully developed turbulent cascade.

In the absence of turbulence, the threshold for dynamo excitation can be determined from knowledge of the steady-state flow geometry. The self-excitation threshold for dynamo action is characterized by the magnitude of the magnetic Reynolds number $R m=V_{0} a / \eta$ (where $\eta=1 / \mu_{0} \sigma$ is the magnetic diffusivity, $V_{0}$ is a characteristic speed of the flow, and $a$ is a characteristic size of the conducting region). We have reconstructed the flow from both direct measurements of the mean flow in an identical device using water (Fig. 2) and from Computational Fluid Dynamics (CFD) simulations. According to kinematic calculations, these flows should exceed the self-excitation threshold at a magnetic Reynolds number of $R m_{\text {crit }} \sim 100^{12,13}$.

Prior measurements of velocity fluctuations in the fully unconstrained geometry have shown that they are well-described as large-amplitude, isotropic, and Gaussian distributed (see Fig. 3) with correlation times on the order of tens of milliseconds ${ }^{11,14}$. The decay time of a dipole field 


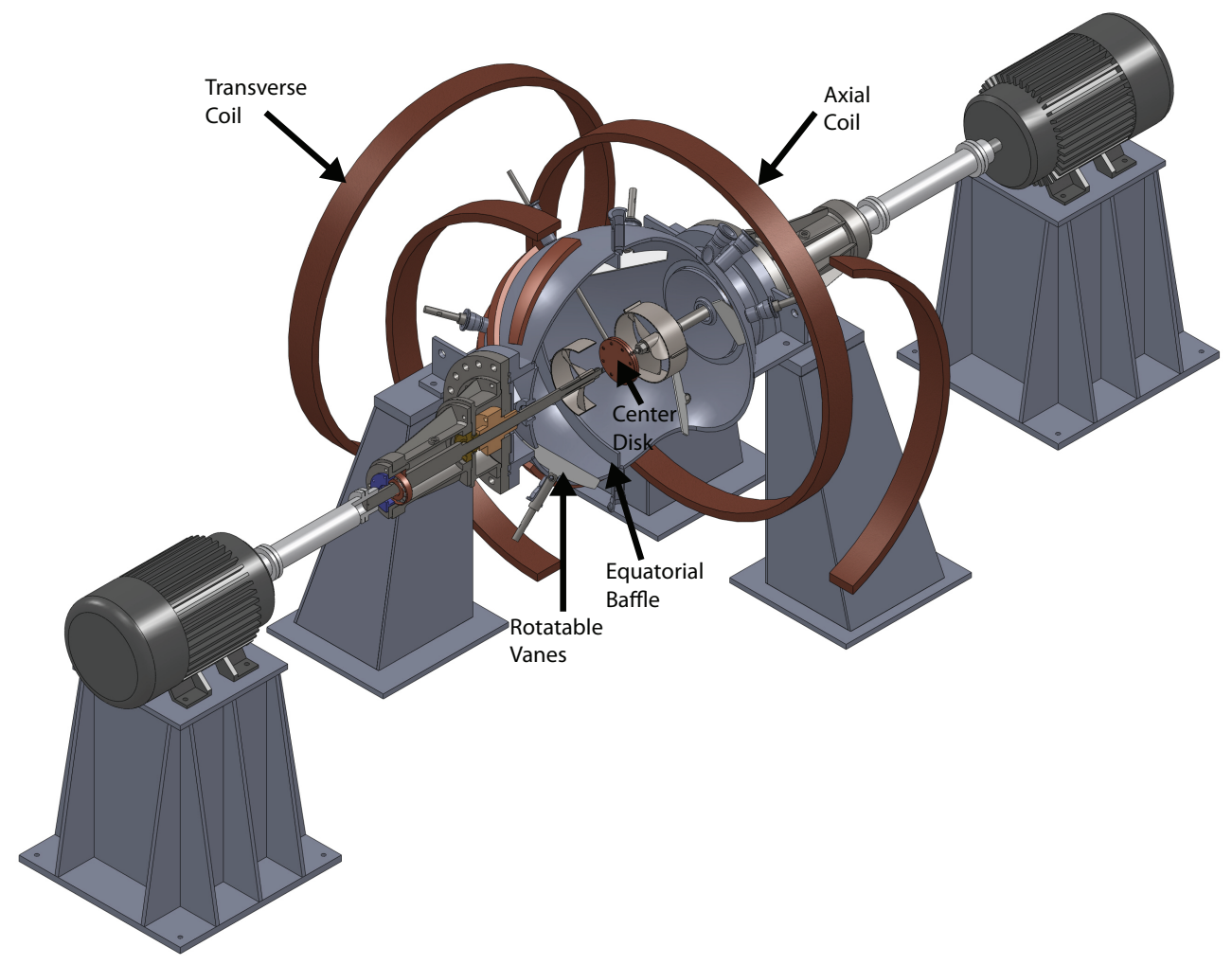

FIG. 1. Schematic of the liquid sodium vessel, impeller assemblies, motors, applied field coils, and boundary flow constraints.

component is estimated as $\tau_{\text {res }}=\mu_{0} \sigma(a / \pi)^{2}=0.3 \mathrm{sec}$. There are no discrete peaks in the power spectrum of the velocity fluctuations; rather, the spectrum is well-described as a Kolmogorov cascade (Fig. 4).

The usual formulation of the mean-field flux turbulent transport coefficients assumes a separation of scales between the mean flow and the fluctuations. In the current experiment, however, the driving scale of the fluctuations is at the same scale length as the mean flow. Also, since liquid metals have a higher resistive diffusion coefficient than viscous diffusion coefficient (characterized by the magnetic Prandtl number $P m=R m / R e \sim 10^{-5}$ ) the scale at which the magnetic field is resistively dissipated is larger than the scale at which eddies are dissipated by viscosity. Since the magnetic fluctuations are heavily damped by resistivity in the wavenumber range $k_{\eta}<k<k_{v}$ the turbulent emf can have no significant amplitude from fluctuations in this range. Hence, the 


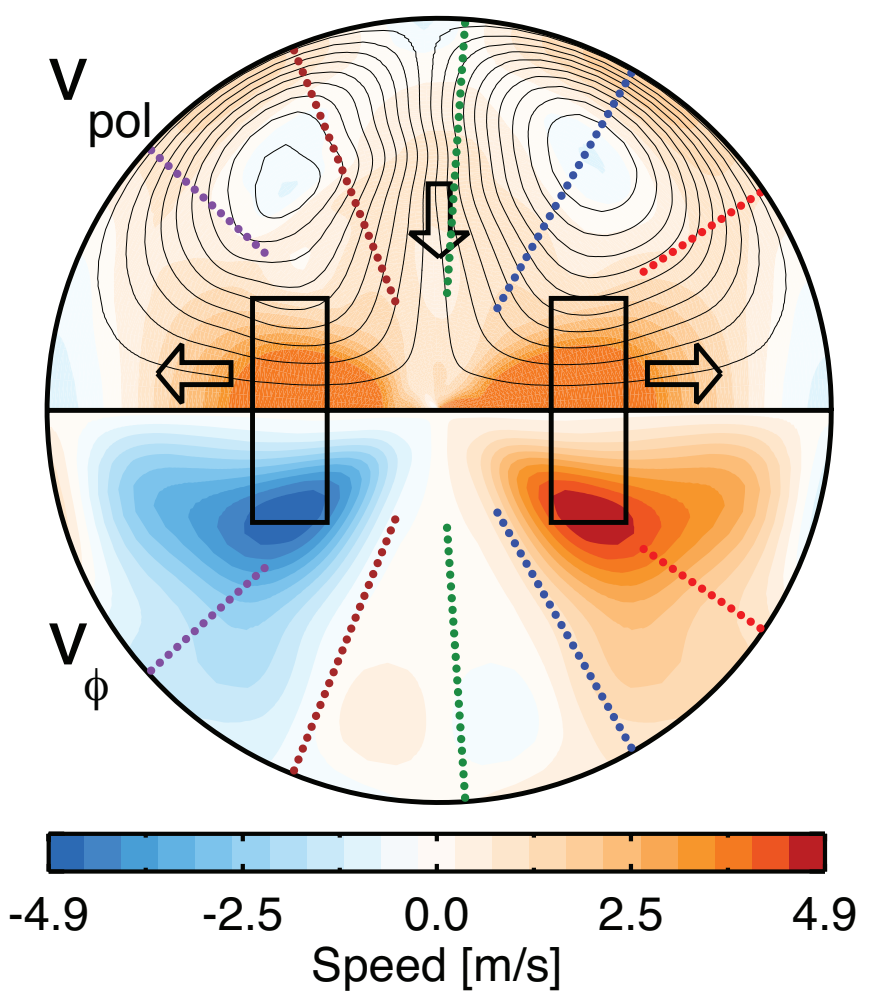

FIG. 2. A contour plot of the mean velocity field reconstructed from Laser Doppler Velocimetry measurements in the water experiment with an unconstrained flow. Measurement locations are indicated by dots.

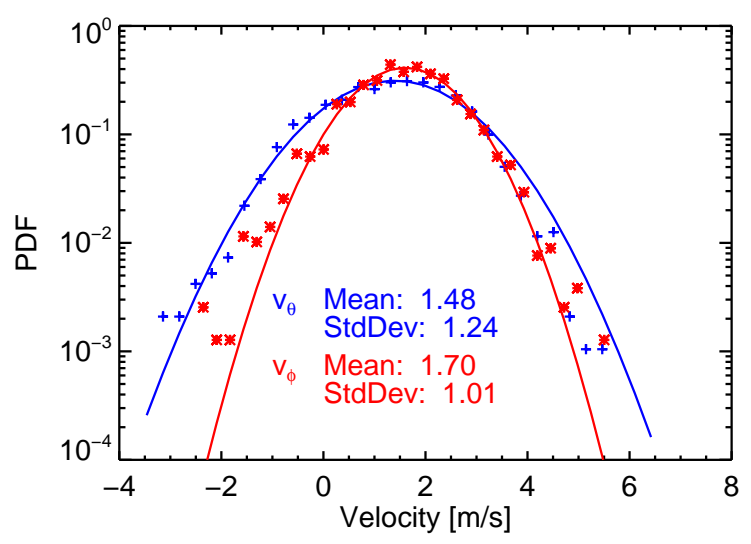

FIG. 3. Probability distribution function of LDV measurements of both $v_{\theta}$ and $v_{\phi}$ located at $r=0.448 \mathrm{~m}$, $\theta=0.60 \mathrm{rad}$ in the unconstrained flow (located just above the region between one of the impellers and the wall). The solid curves are Gaussian curve fits to the distributions. 


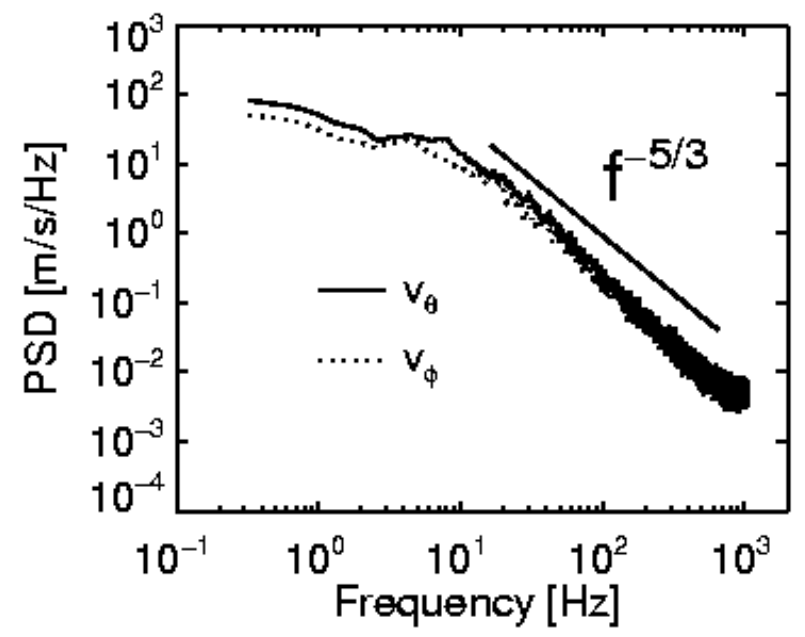

FIG. 4. The velocity power spectral density measurements of $v_{\theta}$ and $v_{\phi}$ at the same location described in Fig. 3 for the unconstrained configuration. The impeller rotation rate is $16.7 \mathrm{~Hz}$. The measurements were taken over the course of $30 \mathrm{~s}$ and so the spectrum is poorly resolved at the lower frequencies. The spectrum is consistent with the $f^{-5 / 3}$ power-law scaling predicted for Kolmogorov turbulence.

only fluctuations capable of contributing to the turbulent emf are between the driving scale and $k_{\eta}$. Since the contributing fluctuations are large scale, they are strongly affected by the boundary conditions of the bulk flow and can be altered by the addition of flow constraints ${ }^{15,16}$. The flow constraints shown in Fig. 1 (Equatorial Baffle, Rotatable Vanes, and Center Disk) were added iteratively to control the symmetry and pitch of the large-scale flow. Although the introduction of the rotatable vanes breaks the azimuthal symmetry, measurements of the magnetic mode spectrum have no significant average $m=3$ component.

\section{MEASUREMENTS OF THE GAIN AND DECAY TIME OF AN APPLIED MAGNETIC FIELD}

The mechanism by which the magnetic field is amplified by advection in the mean flow is illustrated in Nornberg et al. ${ }^{11}$. If a transverse dipole field is applied using the transverse coil set (Fig. 1), we can measure the field induced by the flow using the magnetic sensor array on the surface of the sphere to measure the response magnetic field. The measured magnetic field is a combination of the magnetic field generated by the external coils $\mathbf{B}_{\text {app }}$ and the response magnetic field $\mathbf{B}_{\text {resp }}$ is generated by currents induced by the flowing liquid metal. The external field is 
entirely poloidal; we can best exploit the spherical geometry by decomposing the applied and response magnetic fields into spherical harmonic modes. We will concentrate only on the mode most directly coupled to the transverse coil set which has a $\mathscr{Y}_{\ell=1, m=1}(\theta, \phi)$ angular surface pattern. The transverse dipole portion of the applied and response portions of the magnetic field measured at the surface of the sphere are

$$
\begin{aligned}
\mathbf{B}_{\text {app }}(r=a) & =-S_{1,1}^{\mathrm{app}}(r=a) \mathscr{Y}_{1,1}(\theta, \phi) \hat{r} \\
\mathbf{B}_{\text {resp }}(r=a) & =\frac{2 S_{1,1}^{\text {resp }}(r=a)}{a^{3}} \mathscr{Y}_{1,1}(\theta, \phi) \hat{r} .
\end{aligned}
$$

We define the gain of the electromagnetic system by the ratio of the component of the measured magnetic field co-aligned with the applied magnetic field to the magnitude of the applied magnetic field:

$$
\text { gain }=\frac{S_{1,1}^{\mathrm{app}}(r=a)-2 S_{1,1}^{\mathrm{resp}}(r=a) / a^{3}}{S_{1,1}^{\mathrm{app}}(r=a)} .
$$

We time-average the calculated mode amplitudes from measurements taken at $512 \mathrm{~Hz}$ for three minutes resulting in very small uncertainties on the mean values. Gain measurements for increasing motor speeds and for each of the different baffle configurations are presented in Fig. 5. Before adding constraints, the flow is incapable of significantly increasing the applied magnetic flux. The amplification at higher motor rotation rate increases with each added flow constraint. We find that the boundary modifications effectively reduce the maximum possible correlation length of the velocity fluctuations. Also, the phase angle of the induced field becomes more co-aligned with the applied field as seen in Fig. 6 .

The addition of the rotatable vanes also allows some control of the pitch of the flow which is otherwise determined by the characteristics of the impeller design. The pitch of the impellers, the introduction of a Kört nozzle ring around the impellers (seen in Fig. 1), and the height of the paddles outside the Kört nozzle are all the result of iterative design. Each of these modifications lead to changes to the pitch of the bulk flow with the goal of reducing $R m_{\text {crit }}$. When the equatorial baffle was installed, the fluctuation levels were reduced indicating some stabilization of the shear layer at the equator ${ }^{15}$. At the same time, the pitch of the flow was altered away from optimum. A set of six rotatable copper vanes were installed in the sphere (three in each hemisphere equally spaced in latitude, see Fig. 1). The additions allow for some control over the pitch of the flow as evidenced by the variation in the gain measurements in Fig. 7. As the pitch is varied around $45^{\circ}$ from lines of latitude we find that that amplification of an applied field increases but saturates at a 


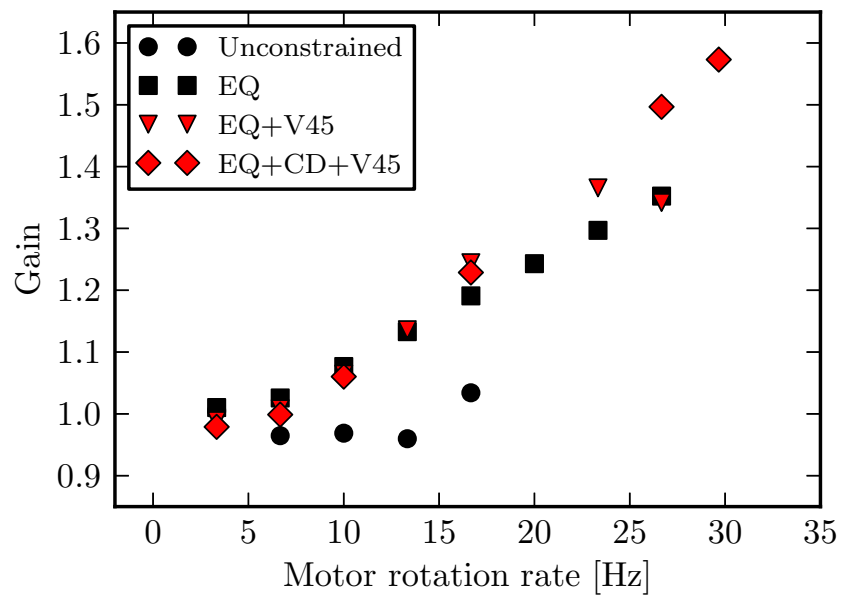

FIG. 5. Magnetic gain measurements as a function of motor rotation rate for the different boundary conditions imposed on the turbulent flow. EQ denotes runs with the equatorial baffle, V45 indicates the angle of the rotatable vanes in degrees with respect to the $\hat{\theta}$-direction, and $\mathrm{CD}$ denotes runs with the copper center disk installed. The unconstraned runs terminate at lower rotation rate due to the higher torque requirements to generate the flows. Introducing baffles greatly reduces the motor load making higher rotation rates possible.

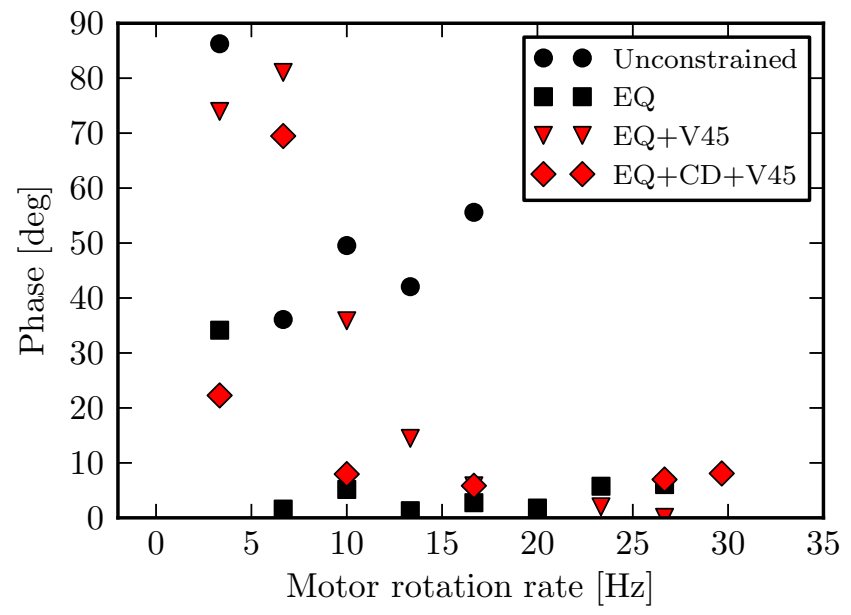

FIG. 6. Phase angle between the $\ell=1, m=1$ components of induced and applied magnetic fields.

lower level than for the case at $45^{\circ}$. One possibility is that the internal magnetic field is amplified sufficiently to apply a new force on the flow. To evaluate this possibility, we quantify the Lorentz force effects by the interaction parameter ${ }^{17} N=\sigma B_{0}^{2} / \rho f_{\text {motor }}$ where $f_{\text {motor }}$ is the motor rotation frequency and $B_{0}$ is conservatively estimated by the maximum measured magnetic field from our internal magnetic sensor array. We find that $N<0.3$ at motor speeds where the gain measurement 


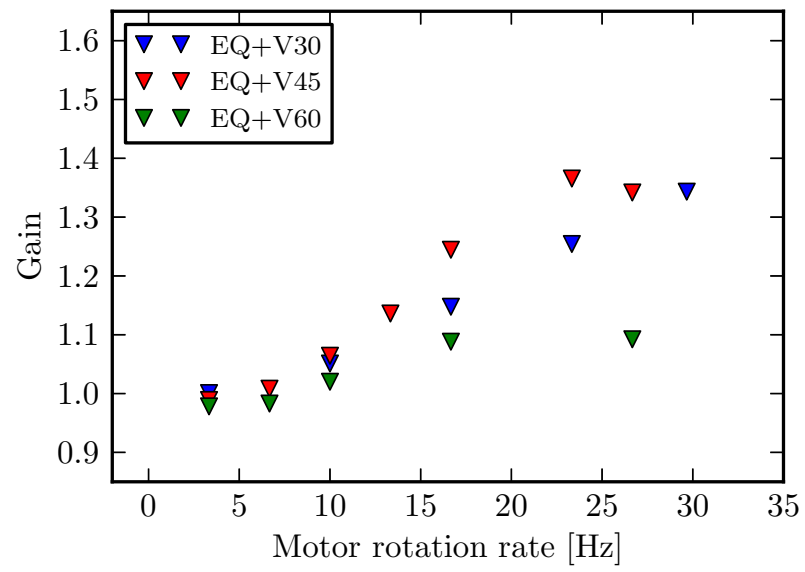

FIG. 7. Magnetic gain measurements as a function of motor rotation rate for variation in the pitch angle of the rotatable vanes.

is saturating suggesting that Lorentz forces are not sufficiently strong to significantly affect the flow.

In addition to steady state applied fields, we performed experiments in which the coils were pulsed to provide several measurements of the decay time of an applied magnetic field. As the flow gets closer to the threshold for exciting a dynamo, we anticipate the decay time of an applied field will lengthen ${ }^{18,19}$. In previous measurements on the Von Kármán Dynamo Experiment, decay times lengthened when the estimated magnetic Reynolds number was within $50 \%$ of the threshold value for a dynamo $R m_{\text {crit }}$. As $R m \rightarrow R m_{\text {crit }}$ the decay times lengthen by a factor of two before a self-generated low amplitude magnetic field is observed ${ }^{18}$. For a nonlinear system with a perfect bifurcation, one can estimate the threshold for instability by extrapolating the measured response to perturbations of the sub-critical state. Attempts to predict $R m_{\text {crit }}$ from linear extrapolation of the measured pulse-decay times result in an over-estimate of $R m_{\text {crit }}$ by $14 \%$ due to the imperfect nature of the bifurcation. Hence, we would anticipate that an extrapolation of the measured sub-critical decay rates would provide an over-estimate of $R m_{\text {crit }}$.

From prior velocity measurements in water, we conclude that the correlation time $\tau_{\text {corr }}$ for the velocity fluctuations is between $0.05-0.5 \mathrm{sec}$ depending on the measurement location. Longer correlation times were observed near the shear layer at the equator due to the primary instability driving the turbulence ${ }^{14}$. After times long compared with $\tau_{\text {corr }}$ the fluctuations are truly independent and identically distributed so that we can construct ensembles of comparable events. To determine the effect of the flow on the decay time of the magnetic field, we pulse the external field 


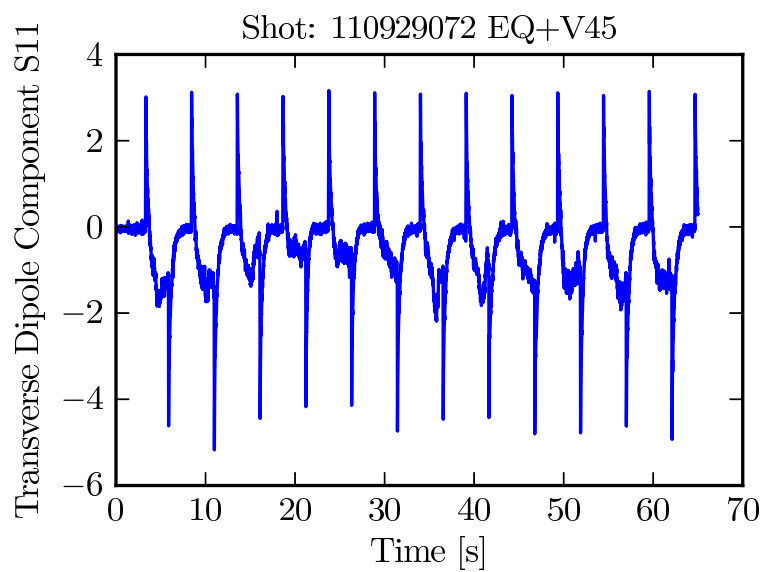

FIG. 8. A time series measurement of the $S_{1,1}(r=a)$ mode of $\mathbf{B}_{\text {resp. }}$. The spikes in the signal are due to the fast switching and are neglected in the pulse decay analysis.

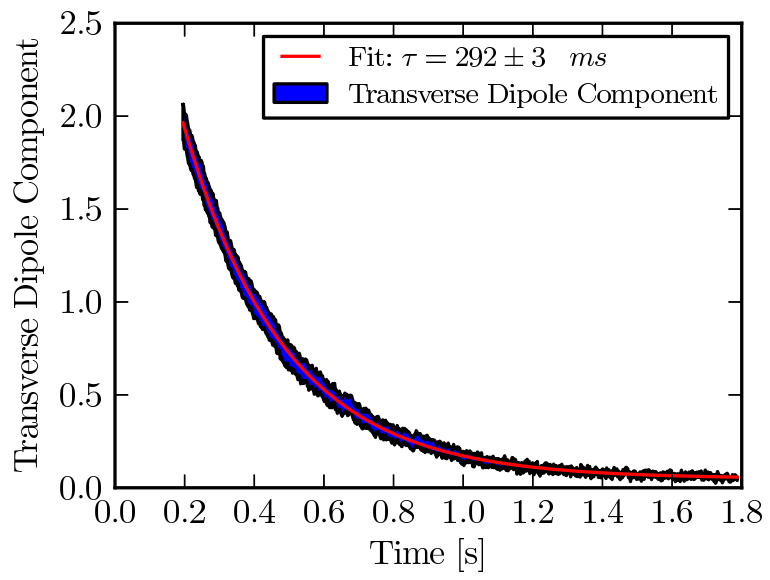

FIG. 9. (bottom) An example ensemble of the decay portion of the measured transverse dipole component for 30 pulses. The times shown are with respect to when the external magnetic field is switched off for each pulse. The shaded area is bounded by the standard deviation of the mean for each point in time which is used at the uncertainty in the fit. The solid line shows the best fit to a decaying exponential obtained by the Levenberg-Marquardt method.

coils at a rate $f_{\text {switch }}=0.2 \mathrm{~Hz}$ so that $f_{\text {switch }} \ll \tau_{\text {corr }}^{-1}$. The response magnetic field is determined by subtracting the applied magnetic field from the measurements and an example is shown in Fig. 8. Each pulse represents an independent measurement of the decay time so we may ensemble the pulses together to reduce the variation due to individual fluctuations before fitting the data to a decaying exponential function as seen in Fig. 9. The deviation of the instantaneous magnetic field measurements from the ensemble average are found to satisfy Gaussian statistics and so we 


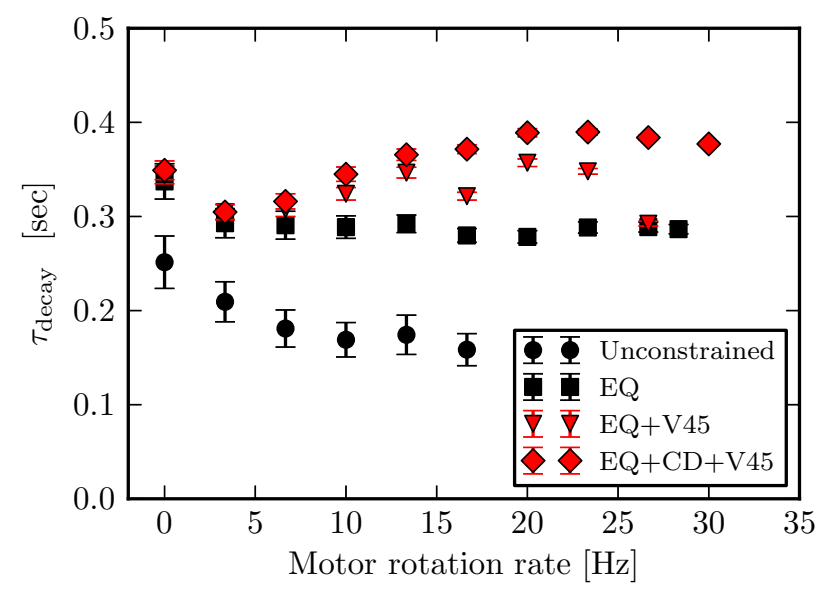

FIG. 10. Pulse decay times for different baffle configurations as a function of the motor rotation rate. Variation of the pulse-decay time for no-flow conditions is associated with temperature variability of the sodium between experiments.

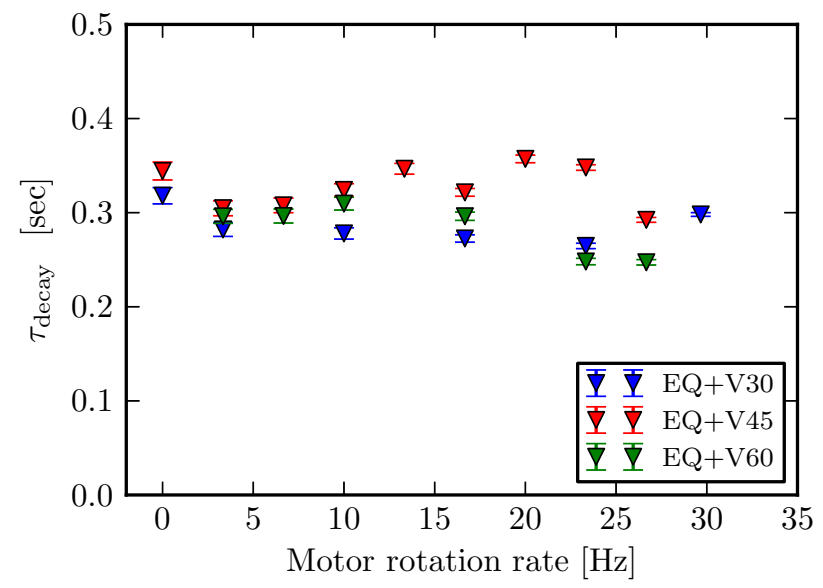

FIG. 11. Pulse decay times measured for runs with the rotatable vanes set to different angles. The variation of the pulse-decay times indicates that the vanes have some effect on the pitch of the flow.

perform a non-linear least squares fit and use the diagonal elements of the covariance matrix to determine the uncertainty in the fit parameters.

What we find from these measurements is that the decay time is lengthened with increasing constraints on the flow and can be modified by changing the pitch of the flow. In the case of a completely free impeller-driven flow, the decay time actually gets shorter than the resistive diffusion time. Increasing the impeller rotation rate has little further effect since added motor power generates more vigorous turbulent eddies which sustain the turbulent rate of diffusion. The longevity of the applied magnetic field is greatly improved when the equatorial baffle is added to constrain 


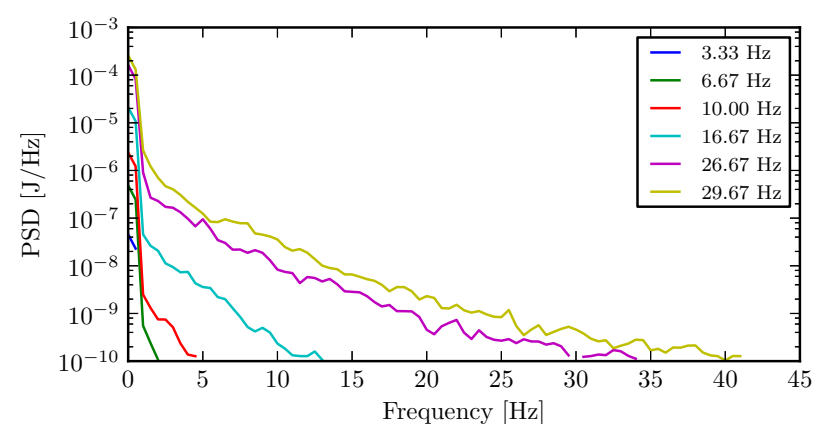

FIG. 12. Power spectral density calculated from the timeseries of the total response field magnetic energy (summed over spherical harmonic modes).

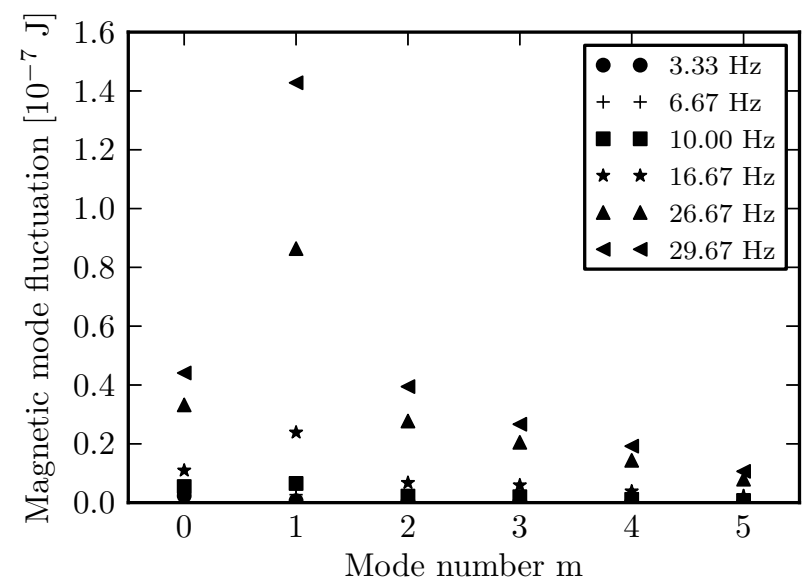

FIG. 13. Response field magnetic energy fluctuations (rms amplitude) calculated in terms of $m$ spherical harmonic mode number (summed over $\ell$ ).

flows that break symmetry across the equator (flows with odd $\ell$ spherical harmonic components). Further improvements are achieved when the rotatable vanes are used to optimize the pitch of the flow as seen in Fig. 11. The final optimization is the introduction of the copper center disk between the two counter-rotating impellers. Although each of these additional flow constraints result in improved performance at lower motor rotation rates, the pulse-decay times are found to saturate at higher rates. The observed magnetic spectrum remains dominated by the $m=1$ component, but there is a significant change in the magnetic fluctuation spectrum. The power spectral density of the measured magnetic energy (summed over all spherical harmonic modes) starts to develop a power law tail as seen in Fig. 12. Likewise, fluctuation power is observed to increase not only in the dominant $m=1$ mode response, but also in the adjacent $m$ modes (Fig. 13).

This saturation raises a caution in extrapolating pulse decay times from a linear fit as shown in 


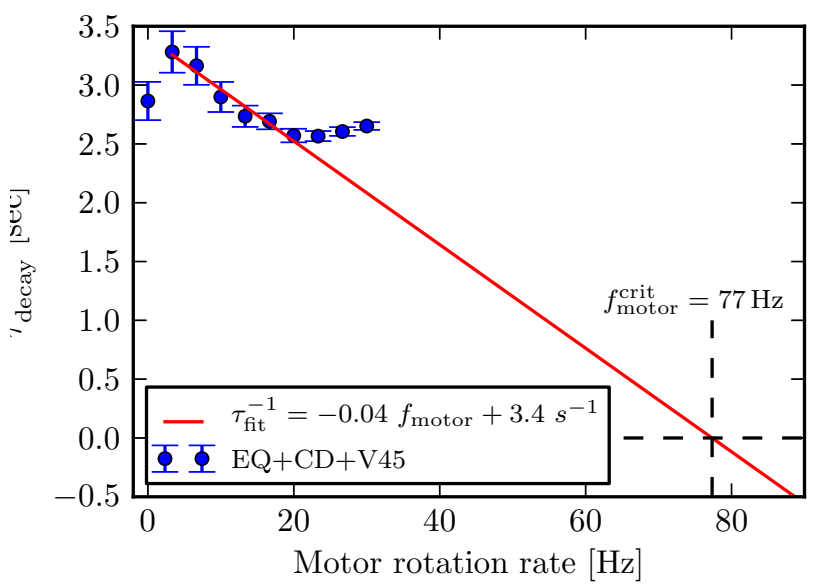

FIG. 14. A plot of the inverse pulse decay times as a function of motor rotation rate for the optimum flow configuration. The first six points which show a clear linear descent are used in a linear fit extrapolated to zero decay rate. The predicted threshold for self-excitation from this technique has a motor rotation rate of $77 \mathrm{~Hz}$ though subsequent measurements show that the decay time reaches a minimum long before this rotation rate.

Fig. 14. Pulse-decay measurements were used in Miralles et al. ${ }^{18}$ to retrospectively predict $R m_{\text {crit }}$ after self-generated fields were observed due to the use of soft-iron impellers. The true transition was found at a lower impeller rotation rate than predicted from the extrapolation of measurements in flows before transition. Here, however, such extrapolation provide a false prediction for eventual dynamo onset due to the resumption of turbulent suppression of the dynamo.

\section{CONCLUSIONS}

Through both direct and indirect measurements, we have characterized the effects of isotropic turbulence on magnetic fields in flow-dominated MHD. The turbulent emf produced by correlated fluctuations is well-described by the mean-field $\beta$-effect which arises as a turbulent enhancement to the fluid resistivity. In hydrodynamic turbulence, it is the largest-scale eddies which give rise to the correlations that transport magnetic field. When those eddies arise at the scale length of the large-scale shear, that is, when the flow lacks scale separation, the amplification and feedback mechanisms of a large-scale slow dynamo are inhibited and the threshold for instability is increased. Moreover, increasing the flow drive further increases the turbulent diffusion so that self-excitation cannot be achieved. 
By reducing the size of the largest eddies, the turbulent resistivity can be significantly reduced. The amplification and feedback of the two-vortex flow are restored so that magnetic flux is more efficiently compressed and twisted. Further constraints to the large-scale flow can improve the amplification and reduce the decay rate of a switched external magnetic field. After significant constraints have been applied to the flow, there is still evidence of turbulent resistive enhancement as motor power is increased and as rotatable vanes controlling the pitch of the flow are varied suggesting that the success in creating self-generated magnetic fields of previous pipe-flow dynamo experiments lies in suppressing the turbulence-induced flows at scales comparable to the flow shear. The reemergence of this turbulent resistivity at higher motor rotation rate makes predictions of the dynamo threshold from sub-critical pulse decay measurements unreliable.

\section{ACKNOWLEDGMENTS}

Special thanks go to Mike Clark and John Wallace who provided engineering support for the baffle modifications. This project is funded by the Department of Energy (DE-FG02-05ER54831)

and is a member experiment of the Center for Magnetic Self Organization in Laboratory and Astrophysical Plasmas (CMSO) (PHY 08-21899). 
*monornberg@wisc.edu

1 K. Rahbarnia, B. P. Brown, M. M. Clark, E. J. Kaplan, M. D. Nornberg, A. M. Rasmus, N. Z. Taylor, C. B. Forest, F. Jenko, A. Limone, et al., Astrophys. J. 759, 80 (2012), URL http://stacks .iop. org $/ 0004-637 X / 759 / i=2 / a=80$.

2 P. K. Kundu and I. M. Cohen, Fluid Mechanics (Academic Press, San Diego, 2008), 4th ed., URL http://library . books24x7. com.ezproxy . library . wisc . edu/toc . asp?bookid=28260.

3 H. K. Moffatt, Magnetic field generation in electrically conducting fluids (Cambridge University Press, Cambridge, England, 1978).

4 K. Krause and K. H. Rädler, Mean-field Magnetohydrodynamics and Dynamo Theory (Pergammon Press, New York, 1980).

5 P. Charbonneau, Living Rev. Solar Phys. 7, 3 (2010).

6 B. Brown, C. Forest, M. Nornberg, E. Zweibel, F. Cattaneo, and S. Cowley, ArXiv e-prints (2011), 1101.0176.

7 A. Gailitis, O. Lielausis, S. Dement'ev, E. Platacis, A. Cifersons, G. Gerbeth, T. Gundrum, F. Stefani, M. Christen, H. Hänel, et al., Phys. Rev. Lett. 84, 4365 (2000).

8 A. Gailitis, O. Lielausis, E. Platacis, G. Gerbeth, and F. Stefani, Rev. Mod. Phys. 74 (2002), URL http://link.aps.org/doi/10.1103/RevModPhys.74.973.

9 R. Stieglitz and U. Müller, Phys. Fluids 13, 561 (2001), URL http://link.aip.org/link/?PHF/ $13 / 561 / 1$.

10 R. Monchaux, M. Berhanu, M. Bourgoin, M. Moulin, P. Odier, J. F. Pinton, R. Volk, S. Fauve, N. Mordant, F. Pétrélis, et al., Phys. Rev. Lett. 98, 044502 (2007), URL http://link.aps.org/doi/10. 1103/PhysRevLett.98.044502.

11 M. D. Nornberg, E. J. Spence, R. D. Kendrick, C. M. Jacobson, and C. B. Forest, Phys. Plasmas 13, 055901 (2006).

12 R. A. Bayliss, C. B. Forest, M. D. Nornberg, E. J. Spence, and P. W. Terry, Phys. Rev. E 75, 026303 (2007), URL http://link .aps.org/abstract/PRE/v75/e026303.

13 K. Reuter, F. Jenko, and C. B. Forest, New Journal of Physics 13, 073019 (2011), URL http: //stacks.iop.org/1367-2630/13/i=7/a=073019. 
14 E. J. Spence, M. D. Nornberg, R. A. Bayliss, R. D. Kendrick, and C. B. Forest, Physics of Plasmas 15, 055910 (2008), URL http://link.aip.org/link/?PHP/15/055910/1.

15 E. J. Kaplan, M. M. Clark, M. D. Nornberg, K. Rahbarnia, A. M. Rasmus, N. Z. Taylor, C. B. Forest, and E. J. Spence, Phys. Rev. Lett. 106 (2011), URL http://link.aps.org/doi/10.1103/ PhysRevLett.106.254502.

16 E. J. Kaplan, B. P. Brown, K. Rahbarnia, and C. B. Forest, Phys. Rev. E 85, 066315 (2012), URL http://link.aps.org/doi/10.1103/PhysRevE.85.066315.

17 M. Bourgoin, R. Volk, P. Frick, S. Khripchenko, P. Odier, and J.-F. Pinton, Magnetohydrodynamics 40, 3 (2004), URL http://mhd.sal.1v/contents/2004/1/MG.40.1.1.R.html.

18 S. Miralles, N. Bonnefoy, M. Bourgoin, P. Odier, J.-F. Pinton, N. Plihon, G. Verhille, J. Boisson, F. Daviaud, and B. Dubrulle, Phys. Rev. E 88, 013002 (2013), URL http://link . aps .org/doi/10.1103/ PhysRevE.88.013002.

19 D. P. Lathrop, W. L. Shew, and D. R. Sisan, Plasma Phys. \& Controlled Fusion 43, A151 (2001). 